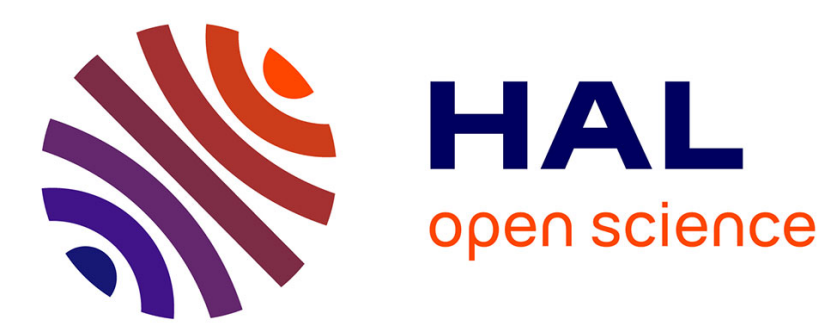

\title{
Dynamic phase-encoding storage of 64 images in a BaTiO3 photorefractive crystal
}

Clara Alves, Gilles Pauliat, Gérald Roosen

\section{To cite this version:}

Clara Alves, Gilles Pauliat, Gérald Roosen. Dynamic phase-encoding storage of 64 images in a BaTiO3 photorefractive crystal. Optics Letters, 1994, 19 (22), pp.1894-1896. hal-00861435

HAL Id: hal-00861435

https://hal-iogs.archives-ouvertes.fr/hal-00861435

Submitted on 12 Sep 2013

HAL is a multi-disciplinary open access archive for the deposit and dissemination of scientific research documents, whether they are published or not. The documents may come from teaching and research institutions in France or abroad, or from public or private research centers.
L'archive ouverte pluridisciplinaire HAL, est destinée au dépôt et à la diffusion de documents scientifiques de niveau recherche, publiés ou non, émanant des établissements d'enseignement et de recherche français ou étrangers, des laboratoires publics ou privés. 


\title{
Dynamic phase-encoding storage of 64 images in a $\mathrm{BaTiO}_{3}$ photorefractive crystal
}

\author{
Clara Alves, Gilles Pauliat, and Gérald Roosen \\ Institut d'Optique Théorique et Appliquée, Unité de Recherche 14 Associée au Centre National de la Recherche Scientifique, \\ Centre Scientifique, Bâtiment 503, B.P. 147, 91403 Orsay Cedex, France
}

Received April 22, 1994

Storage of 64 images $(128 \times 128$ pixels $)$ by the deterministic phase-encoding technique in a holographic memory is reported. A ferroelectric phase modulator permits a fast random image reconstruction ( $150 \mu \mathrm{s} / \mathrm{image})$. Sources of cross talk arising from the imperfections of the optical components are studied and evaluated.

We present a photorefractive (PR) memory setup that permits the storage of $N=64$ images of $128 \times 128$ binary pixels in a $\mathrm{BaTiO}_{3}$ crystal by the deterministic phase-encoding technique. ${ }^{1-4}$ Because of the absence of any moving mechanical parts this multiplexing method permits a reliable and very fast random access to the images. This memory could be used in digital computing or for pattern recognition. ${ }^{1}$

This technique consists of recording the $N$ images by interference of the image beam with all $N$ reference beams. These beams are angularly spaced by more than the Bragg angle so that each of them reconstructs the holograms it has written. The same $N$ reference beams are used to record all images. However, the set of relative phase $(0$ or $\pi)$ between the reference beams and the image beam is changed for each image and represents the image address. We reconstruct the images by reading out the holograms with the entire set of reference beams carrying the phase code corresponding to the image address. Thus, during image retrieval, all the $N$ reconstructed beams for each of the $N$ images interfere. If a set of orthogonal binary (i.e., 0 and $\pi$ ) Walsh-Hadamard phase $\operatorname{codes}^{2-5}$ is chosen, all reconstructed beams corresponding to that image interfere constructively, while all others interfere destructively. However, in practice, incomplete destructive interferences produce cross talk. We previously ${ }^{5}$ found the two following sources of cross talk, which were evaluated for binary stored images with the same probability for white and black pixels: (i) The phase modulator introduces a phase shift of 0 or $\pi+\varepsilon$ on each beam. The predominant source of cross talk arises when the phase-shift error $\varepsilon$ is the same on all $N$ reference beams (systematic error). This is the only source that we consider here. In that case the amplitude signal-to-noise ratio (SNR) limited by cross talk was found to be

$$
\mathrm{SNR}_{\mathrm{amp}} \approx 2 /[(N-1) \varepsilon] .
$$

(ii) The amplitudes of all $N$ diffracted beams corresponding to the same image are not identical. If the amplitude of these beams is a random variable with mathematical expectation $\langle u\rangle$ and variance ${\sigma_{u}}^{2}$, then the noise induced by cross talk is also a random vari- able. We found that the probability for $\mathrm{SNR}_{\mathrm{amp}}$ to be larger than $r_{0}$ is

$$
\begin{array}{r}
\operatorname{Prob}\left(\mathrm{SNR}_{\mathrm{amp}}>r_{0}\right)=\frac{2}{\sqrt{2 \pi}} \int_{0}^{1 / r_{0} \beta} \exp \left(-t^{2} / 2\right) \mathrm{d} t, \\
\text { with } \beta^{2}=\frac{(N-1)}{2 N}\left(\frac{\sigma_{u}}{\langle u\rangle}\right)^{2} .
\end{array}
$$

Our goal is to make this probability close to unity for $r_{0}$ equal to the amplitude contrast of the imput-image spatial light modulator (SLM).

We have optimized our memory setup, trying to minimize these two sources of cross talk, and describe the solutions that we found to achieve large SNR. The setup is shown in Fig. 1. The laser beam $(532 \mathrm{~nm})$ is divided between an image arm and a reference arm. A computer-generated fan-out hologram (CGH) diffracts the incident beam in $N$ reference beamlets that are individually phase modulated. All the beams interfere in a $\mathrm{BaTiO}_{3}$ crystal. We also describe the realization and characterization of the phase modulator and explain the entire reference arm and the choice of the crystal, and then we present the image arm. Finally, we characterize the memory.

A $0-\pi$ phase modulation can be achieved with a binary-polarization-only SLM sandwiched between two crossed polarizers. ${ }^{6}$ The SLM acts as an array of $N$ half-wave plates. Each input beam passes through one of these pixels. The beams are linearly polarized along the bisector of the two possible states of the fast axis of the wave plates. After passing through a wave plate the beams have one of two pos-

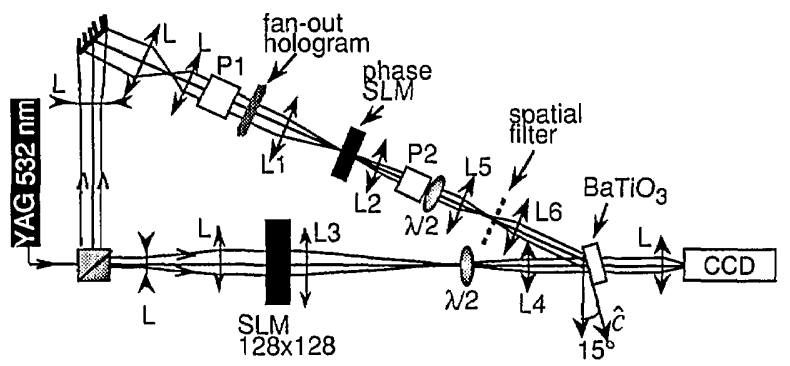

Fig. 1. Experimental setup for 64-phase multiplexed hologram storage. $\mathrm{P}_{1}, \mathrm{P}_{2}$, polarizers; L's, lenses; $\lambda / 2$ 's, half-wave plates used for making the incident beams extraordinarily polarized. 
sible linear polarizations. An output polarizer set at $90^{\circ}$ to the input polarization state transforms the polarization modulator into a phase modulator. For our realization we choose a $10 \times 10$ pixel ferroelectric polarization SLM from Displaytech. The entire array can be reconfigured in $150 \mu \mathrm{s}$, which corresponds to the memory image access time. Only 64 pixels are used here to modulate the 64 reference beams.

In principle such a phase modulator gives a perfect $\varphi_{\text {ph.mod. }}=\pi$ phase shift; however, it suffers from three main defects. The first comes from an imperfect crossing of the two polarizers (they are crossed at $\pi / 2+\delta$ ). The second arises from the phase birefringence $\psi_{\mathrm{w} \text {.plate }}$ of the wave plate, which may be different from $\pi$. The third defect results from an imperfect alignment (angle $\gamma \neq 0$ ) of the input polarizer along the bisector of the two possible states of a waveplate fast axis. The optical axis of the ferroelectric liquid crystal rotates through $\sim 45^{\circ}$. To evaluate the importance of these imperfections, we calculate the optical electric field transmitted by the phase modulator in both states. At first order in $\gamma$ and $\delta$ we find the expression for the modulation of the amplitude of the transmitted beam:

$$
\sigma_{u}^{\text {ph.mod. }} /\langle u\rangle=\left(\left|u_{1}\right|-\left|u_{2}\right|\right) /\left(\left|u_{1}\right|+\left|u_{2}\right|\right) \approx 2 \gamma+\delta .
$$

Phase error $\varepsilon$ is of second order in $\delta\left(\psi_{\text {w.plate }}-\pi\right)$ :

$$
\varepsilon \approx \sqrt{2} \delta \sin \left(\psi_{\text {w.plate }}-\pi\right) .
$$

Relation (4) demonstrates that a very accurate $\varphi_{\text {ph.mod. }}=\pi$ phase shift is easily obtained. Because we use only one polarizer and one analyzer for the entire array of pixels we first check that the liquidcrystal alignment is the same for all pixels within our measurement accuracy $\left(|\zeta| \leq 3 \times 10^{-3} \mathrm{rad}\right.$ in each of the states).

We cross the polarizers with a precision better than $|\delta| \leq 3 \times 10^{-3} \mathrm{rad}$, and we estimate $\psi_{\text {w.plate }}$ by measuring the intensity modulation of the output wave, aligning the first polarizer along one optical axis and the second so that the contrast is maximum. We find that $\psi_{\text {w.plate }}=\pi+0.15 \mathrm{rad}$. Therefore from relation (4) we find that the error in the phase modulation for all the pixels is lower than $|\varepsilon| \leq 6 \times 10^{-4} \mathrm{rad}$. Thus the ferroelectric phase SLM is very accurate, and compared with a nematic one it is much faster, with a resulting phase modulation that is temperature independent. From relation (1) we deduce that the $\mathrm{SNR}_{\mathrm{amp}}$ limited by $\varepsilon$ is larger than 53 (i.e., the intensity SNR is larger than 2800), and from Eq. (3) we find that the amplitude modulation induced by the phase modulator for all transmitted beams is lower than $2 \zeta+\delta \leq 1 \%$. Such an amplitude modulation cannot be detected in our experiment. As shown below, other sources of amplitude variations among the reference beams predominate.

A CGH splits a single beam into an array of $\sqrt{N} \times$ $\sqrt{N}$ reference beams of equal intensity. ${ }^{7}$ All diffracted amplitudes are equal to within $\sigma_{u}^{\text {holo. }} /\langle u\rangle \approx 4 \%$. The hologram is Fourier transformed by lens $L_{1}$ in Fig. 1, producing a square array of $\sqrt{N} \times \sqrt{N}$ spots that coincides with the pixel array of the phase-only SLM (pixel pitch $p=1 \mathrm{~mm}$ ). These spots are then inverse Fourier transformed by lens $\mathrm{L}_{2}$ (focal length $f_{2}=31 \mathrm{~mm}$ ). All reference beams thus overlap in the image focal plane of that lens, which is also the image of the CGH. This focal plane is then imaged onto the PR crystal by means of another refractive telescope ( $4 f$ system of $\mathrm{L}_{5}$ and $\mathrm{L}_{6}$ ). All the $N$ reference beams thus overlap the image beam in the crystal. An array of 64 holes inserted in the middle of this last telescope acts as a spatial filter, stopping the zero and second undesirable orders produced by the CGH.

To prevent cross talk, each reference beam must reconstruct only those holograms that it has recorded. A grating recorded by a reference beam and by an image beam must not be read out by another reference beam. We thus take advantage of the Bragg selectivity, which exists in the incident plane only (along the $\theta$ direction defined by the image beam wave vector and the average reference beam wave vector). In the orthogonal direction $\phi$, no selectivity exists because of the Bragg degeneracy. ${ }^{8}$ The angular width of the reference beam matrix is $7 p / n f_{2}$, where $n=2.4$ is the crystal refractive index. If the phase SLM is rotated by an angle $\omega$ around the axis of light propagation, we can use the Bragg selectivity as follows: We choose $\omega=0.1 \mathrm{rad}$ so that the spacing $A_{\theta}=p \sin (\omega) / n f_{2}$ between two reference beams along the $\theta$ direction is larger than the Bragg selectivity $\Delta \theta_{B}$. With the crystal and the orientation that we use, we indeed have $\Delta \theta_{B} \approx 8 \times 10^{-4} \mathrm{rad}$. We then check that the upper reference beam wave vector of one column does not overlap the lower reference beam wave vector of neighboring columns within the Bragg selectivity. The SNR limited by energy diffracted from non-Bragg-matched gratings is therefore very high, ${ }^{9}$ and we do not take it into account.

The $\mathrm{PR}$ medium is a $\mathrm{BaTiO}_{3}$ crystal whose size is $3.63 \mathrm{~mm} \times 5.24 \mathrm{~mm} \times 5.22 \mathrm{~mm}$ along the $\hat{a} \times \hat{b} \times \hat{c}$ crystallographic axes. It is chosen for its high achievable index modulation and for its long-term storage, which is more than one day in the dark. The input beams are extraordinarily polarized and enter by the $\hat{a}$ face. The refractive angle of the image beam is $\theta_{\mathrm{im}} \approx 6^{\circ}$, and the average refractive angle of the reference beams is $\theta_{\text {ref }}=17^{\circ}$. Because for this configuration the fringe spacing is much larger than the Debye screening length, the induced refractive-index modulation varies approximately linearly with the refractive angle of the reference beams. This index modulation variation $v \approx \pm 17 \%$ induces differences between the amplitude diffraction efficiencies that correspond to the following variance: $\sigma_{u}^{\text {crsytal }} /\langle u\rangle \approx 10 \%$. A $128 \times 128$ binarypixel ferroelectric SLM (from CRL) produces the 64 images to record. The image of this SLM is projected in the crystal by field lens $\mathrm{L}_{3}$ and imaging lens $L_{4}$. With this arrangement the phase defects of the crystal remain invisible in the retrieved amplitude image.

The images are recorded with the incremental procedure. $^{2}$ The image beam intensity is approximately one half of the total reference beam intensity, 

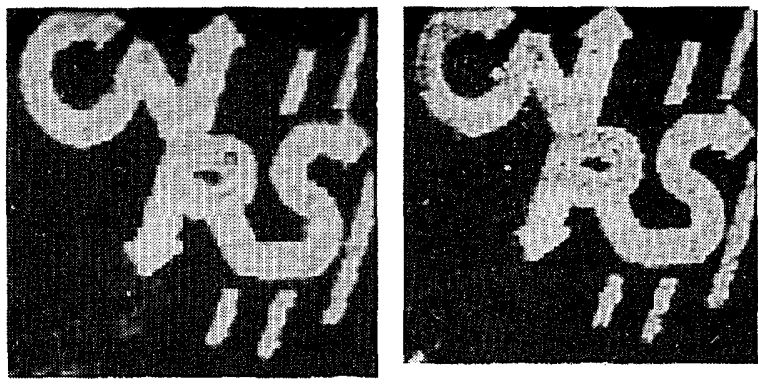

Fig. 2. One of the 64 images transmitted before storage (left-hand side) and retrieved (right-hand side).

and the intensity diffraction efficiency for entirely white images is $\eta=2.5 \times 10^{-4}$. More complex images are also recorded (see Fig. 2). The access time to retrieve any of the images is $150 \mu \mathrm{s}$. For a 3$\mathrm{mW} \mathrm{cm} \mathrm{cm}^{-2}$ total incident intensity the time required to record all 64 images to saturation is a few minutes (PR time constant is $\sim 1 \mathrm{~min}$ ). To study the cross talk in the retrieved images, we record $64 \mathrm{im}$ ages with 63 little black squares and 1 white square. The pixel position differs in each image. In this configuration we expect very little cross talk because it comes only from the superpositon of one white pixel onto 63 black ones. The intensity contrast for the transmitted images that we measured on a CCD camera before recording is $\sim 40: 1$ (the contrast limitation is given by the $128 \times 128 \mathrm{SLM}$ ). Once the images are stored in the crystal, the intensity contrast of the retrieved images decreases to 20:1 (i.e., the amplitude contrast is $C_{\text {ampl. }} \approx 4.5$ ). This contrast reduction is expected; it arises from the nonlinear dependence of the interference pattern modulation depth on the image-to-reference intensity ratio and thus on the recorded hologram modulation.

We have seen above that the cross talk induced by the imperfect phase modulation is totally negligible. The main source of noise in the retrieved images thus originates from the nonuniformity of the diffracted reference beams. The fan-out hologram, the phase modulator, and the index modulation variation of the PR grating contribute to this nonuniformity. The total standard deviation of this amplitude nonuniformity is equal to

$$
\left[\left(\frac{\sigma_{u}^{\text {holo. }}}{\langle u\rangle}\right)^{2}+\left(\frac{\sigma_{u}^{\text {ph.mod. }}}{\langle u\rangle}\right)^{2}+\left(\frac{\sigma_{u}^{\text {crystal }}}{\langle u\rangle}\right)^{2}\right]^{1 / 2} \approx 11 \% .
$$

From Eq. (2) we find that the probability for the SNR limited by this nonuniformity to be larger than the contrast of the received images is $\operatorname{Prob}\left(\mathrm{SNR}_{\mathrm{amp}}>\right.$ $4.5) \approx 0.996$. Therefore the cross talk induced by the experimental setup imperfections should not be visible with the image SLM.

To check that point, we record 64 images with a white background and a single black pixel. The location of this black pixel changes for each image. If there is cross talk, the black pixel should become white because at its site all the other images are white. This corresponds to the worst situation, with a noise larger than that computed from Eq. (2). We find that the contrast does not change, and we again measure an intensity contrast of $20: 1$.

To compare the theoretical predictions of Eq. (2) with experiments, we compute from Eq. (2) and relation (5) the mean value of the intensity SNR and we find that $\mathrm{SNR}_{\text {int }}=165$. To measure the ultimate $\mathrm{SNR}_{\text {int }}$ we use a mechanical shutter to produce entirely white or black images to fulfill the calculation hypothesis of equiprobable white and black pixels and measure the diffraction efficiency with a photodiode in place of the CCD camera. We find that $S_{N R}$ int $\approx 140$, which is in good agreement with the computed value and demonstrates that the cross talk induced by imperfections of optical components is totally negligible in our setup.

Our setup characterization shows that we are still far away from reaching the limitations of the deterministic phase-encoding technique. For instance, by use of a phase modulator of the same quality as the one we have today but with a larger number of pixels, more than 500 -images could be stored, while an intensity SNR larger than $40: 1$ is maintained. A further increase in capacity could be gained by insertion of a spatially uniform phase modulator in the image beam, as described in Ref. 5. The cross talk induced by nonuniform diffracted amplitudes does not directly depend on the number of stored images, except through the index modulation variation $v$. We may reduce it by bringing the reference beams closer; they are indeed separated by $2 \Delta \theta_{B}$, whereas $\Delta \theta_{B}$ is sufficient. We can also use PR crystals with special cuts, ${ }^{10}$ permitting us to reach the maximum $\mathrm{PR}$ index modulation while minimizing the index variation $v$. In view of these results we believe that fast-access memories with image capacities much larger than 64 may be easily obtained.

This study was conducted in the framework of the Parallel Optical Processors and Memories project.

\section{References}

1. C. Alves, P. Aing, G. Pauliat, and G. Roosen, Proc. Soc. Photo-Opt. Instrum. Eng. 48, 221 (1993).

2. Y. Taketomi, J. E. Ford, H. Sasaki, J. Ma, Y. Fainman, and S. H. Lee, Opt. Lett. 16, 1774 (1991).

3. C. Denz, G. Pauliat, G. Roosen, and T. Tshudi, Opt. Commun. 85, 171 (1991).

4. J. Lembcke, C. Denz, T. H. Barnes, and T. Tschudi, in Photorefractive Materials, Effects, and Devices, Vol. 14 of 1991 OSA Technical Digest Series (Optical Society of America, Washington, D.C., 1991), p. 574.

5. C. Denz, G. Pauliat, G. Roosen, and T. Tschudi, Appl. Opt. 31, 5700 (1992).

6. D. Psaltis, E. G. Paek, and S. S. Venkatesh, Opt. Eng. 23, 698 (1984).

7. Parallel Optical Processors and Memories (POPAM) project funded by Commission of the European Community under the ESPRIT Program for Research and Development.

8. H. Lee, X. Gu, and D. Psaltis, J. Appl. Phys. 65, 2191 (1989).

9. K. Curtis and D. Psaltis, J. Opt. Soc. Am. A 10, 2547 (1993).

10. J. E. Ford, Y. Fainman, and S. H. Lee, Appl. Opt. 28, 4808 (1989). 